\title{
Transatlantica
}

Revue d'études américaines. American Studies Journal

\section{La migration en héritage dans les Amériques}

6-7-8 juin 2012

Hilary Sanders

\section{(2) OpenEdition}

Journals

Édition électronique

URL : https://journals.openedition.org/transatlantica/5887

DOI : 10.4000/transatlantica.5887

ISSN : $1765-2766$

Éditeur

Association française d'Etudes Américaines (AFEA)

Référence électronique

Hilary Sanders, «La migration en héritage dans les Amériques », Transatlantica [En ligne], 2 | 2012, mis en ligne le 23 mai 2013, consulté le 06 avril 2023. URL : http://journals.openedition.org/transatlantica/ 5887 ; DOI : https://doi.org/10.4000/transatlantica.5887

Ce document a été généré automatiquement le 6 avril 2023.

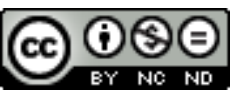

Creative Commons - Attribution - Pas d'Utilisation Commerciale - Pas de Modification 4.0 International - CC BY-NC-ND 4.0

https://creativecommons.org/licenses/by-nc-nd/4.0/ 


\title{
La migration en héritage dans les Amériques
}

\author{
6-7-8 juin 2012
}

Hilary Sanders

1 Le $10^{\mathrm{e}}$ colloque annuel de l'Institut des Amériques, animé par l'Université Paris Diderot - Paris 7, avait comme thème cette année le phénomène migratoire dans les deux continents américains. Comme le rappelle le texte de cadrage, le "caractère constitutif» des migrations dans la construction des pays du "Nouveau Monde» justifie le choix de ce sujet transversal, devenu un objet d'étude privilégié des sciences sociales au cours du $20^{\circ}$ siècle. Coordonnés par Françoise Lestage et Paul Schor, ces trois jours de conférences ont réuni sociologues, historiens, anthropologues, géographes, politologues, et civilisationnistes. Les chercheurs étant venus de toute l'Europe, de l'Amérique du nord et de l'Amérique du sud, le colloque s'est déroulé en trois langues, à l'aide de traductions simultanées.

2 Le comité d'organisation a choisi de préciser la vaste thématique que représente l'immigration en invitant les participants à aborder la transmission, ou "l'héritage » des phénomènes étudiés et à "placer résolument le migrant au centre de l'analyse ». Alors que cette première contrainte a été respectée de manière variable selon les interventions, la deuxième a clairement inspiré des contributions basées sur des études empiriques et très spécifiques. Étant donné que ces communications s'étendaient en outre sur cinq siècles d'histoire et une aire géographique comportant 22 pays, elles ont été organisées selon des sous-thématiques larges et inclusives: temporalités, trajectoires, expériences et transmissions. Trois conférences plénières ont aussi figuré dans le programme.

3 L'historienne Mae Ngai, auteure d'Impossible Subjects: Illegal Aliens and the Making of Modern America, a inauguré le premier jour du colloque avec une conférence critiquant le récit populaire des États-Unis comme une "nation d'immigrants ", un récit qui fait abstraction des histoires parallèles de la conquête des territoires amérindiens et mexicains, de l'esclavage, des projets impérialistes et d'une longue interdiction de l'immigration asiatique. Mae Ngai a établi dans un premier temps que l'émigration 
européenne n'est perçue comme une immigration d'étrangers qu'à la fin du $19^{\mathrm{e}}$ siècle, lorsque l'origine et la religion des nouveaux arrivants suscitent un rejet de la part des «natifs ». Ensuite, ce sentiment xénophobe domine les politiques migratoires et influe sur l'étude de l'immigration pendant un demi-siècle. Une historiographie qui interprète le phénomène comme un élément fondateur de la nation, plutôt qu'un problème social, émerge seulement dans la période de l'après-guerre. Ce changement de paradigme, ainsi que l'ouverture politique à l'immigration qui s'ensuit, serait un produit de l'ascension socio-économique des générations issues des grandes vagues migratoires du début du siècle. Mae Ngai suggère que, malgré l'absence d'un contexte économique aussi favorable, les tendances démographiques actuellement à l'œuvre - notamment l'accroissement de la population «Latino » - aboutiront néanmoins à une prise de pouvoir similaire de la part des descendants des migrants contemporains, aujourd'hui marginalisés, exploités et expulsés en masse.

Moins politique dans l'ensemble que l'intervention de Mae Ngai (anciennement militante des droits des sans-papiers à New York), la majorité des communications dans les ateliers du colloque impliquait d'ailleurs une notion de la "migration" prise au sens large. Les exemples de parcours individuels ou collectifs des migrants ont abordé une multiplicité de types de mobilités, dont l'expérience des réfugiés, des touristes, des universitaires, des missionnaires, des militants et des mères de migrants disparus. Comme l'a fait remarquer la sociologue Jocyline Streiff-Fénart, des points communs existaient néanmoins dans la navigation par ces migrants entre les opportunités offertes par des réseaux informels et les dispositifs d'aide ou de contrôle institutionnel auxquels ils étaient confrontés. Certaines communications ont exploré la négociation de la part des migrants avec un contexte d'accueil plus ou moins hostile; Laura Hobson Faure (Université Paris 3 - Sorbonne Nouvelle) a exposé ses recherches actuelles sur des enfants français de confession juive envoyés aux États-Unis pendant la Deuxième Guerre mondiale, en dépit du système restrictif des quotas en vigueur. Suivant le témoignage d'un cas particulier, elle a décrit la politique assimilationniste des organisations d'aide aux réfugiés qui plaçaient ces enfants dans des familles d'accueil dispersées à travers le pays. Magdalena Barros Nock (CIESAS, Mexique), pour sa part, a présenté l'expérience d'un groupe d'adolescents mexicains autochtones dans une zone rurale de la Californie, confrontés au racisme de leurs compatriotes mestizos.

D'autres communications donnaient à voir une vision plus positive de l'intégration des migrants. La sociologue Kristen Lucken (Brandeis University, États-Unis) a postulé que la religion Hindi telle qu'elle est pratiquée parmi la deuxième génération de migrants indiens permet d'affirmer leur inclusion dans le modèle pluraliste défendu dans la Constitution américaine, malgré la domination de la foi chrétienne. Prenant comme objet d'étude "l'ancrage » des cubains à Miami depuis les années 1960, la géographe Violaine Jolivet (Université de Montréal) a exploré les stratégies migratoires, résidentielles et politiques qui ont fabriqué et transformé un espace urbain où un habitant sur trois est d'origine cubaine. Ce concept d'ancrage, défini comme un processus dynamique qui doit être appréhendé en référence à la mobilité, a été particulièrement apprécié par le public.

6 La notion de l'héritage ou de la mémoire des migrations s'est manifestée dans les communications à travers l'analyse d'une variété de sources destinées à transmettre ce phénomène, dont des entretiens, des photographies, des lettres, des journaux intimes, des expositions institutionnelles et des archives administratives. L'influence du présent 
sur la construction de la mémoire du passé est un aspect de la transmission de l'expérience migratoire abordé par plusieurs intervenants. Dominique Daniel (Oakland University, Michigan) a exploré les historical societies qui apparaissent à la fin du $19^{\mathrm{e}}$ siècle, créées par des groupes juifs, allemands et finlandais dans le but de réunir des archives qui racontent l'histoire du groupe aux États-Unis. Une professionnalisation de la mémoire s'observe dans ces institutions dont les dirigeants sont souvent soucieux de faire preuve d'une intégration réussie à la société américaine. Audrey Célestine (Université Lille 3), à partir des recherches sur le Center for Puerto Rican Studies à New York, a également parlé de la formation d'un récit collectif qui présente une identité unifiée d'un groupe ethnique. Elle identifie, à travers les archives mises en avant par le centre, un certain «lissage» des différentes mobilisations des portoricains, dont certaines comportaient des revendications plus radicales, aujourd'hui occultées. Dans ses remarques à propos de l'ensemble du colloque, l'historienne Nancy Green (EHESS) a aussi perçu dans certaines sources étudiées une version romancée de la migration qui tait des aspects moins valorisants de l'expérience. Alors que certaines communications se limitaient aux paroles et aux images laissées, elle avance que ce qui manque dans les auto-représentations des migrants et de leurs descendants, par oubli ou par omission consciente, mérite parfois autant d'analyse.

7 Le cadrage et l'interprétation des divers types de sources examinées dans les communications ont par ailleurs donné à voir des différences méthodologiques frappantes entre des chercheurs formés par les normes de leur discipline et de leur milieu universitaire national. Dans ce contexte interdisciplinaire, un clivage entre une approche historique traditionnelle, axée sur les grandes tendances sociétales, et celle des chercheurs «de terrain» était particulièrement visible. Ramón Gutierrez (Université de Chicago) a rappelé que l'étude des productions écrites et des prises de paroles individuelles oriente la recherche vers les élites qui produisent plus de documents et verbalisent plus facilement leur expérience que les classes sociales défavorisées. Ainsi, comme l'a souligné l'historien Fernando Devoto (Université de Buenos Aires), l'analyse de l'expérience du migrant risque d'occulter celle des migrants, cette masse anonyme qui laisse peu de traces. Pourtant, une quête de mémoire de la part de la masse peu influente d'anciens Braceros - «des oubliés de l'histoire contemporaine mexicaine »- était l'objet de recherche de Philippe Schaffhauser (COLMICH, Mexique), qui a expliqué comment les tentatives de reconstruire une mémoire familiale est en partie à l'origine du combat de ces travailleurs et de leurs descendants pour faire valoir leurs droits à une pension de la part des États-Unis.

Lævenue d'Aristide Zolberg (New School for Social Research, New York), auteur de l'ouvrage magistral A Nation by Design: Immigration Policy in the Fashioning of America, était particulièrement attendue lors du dernier atelier du colloque. Il a interrogé dans sa communication les particularités de l'incorporation - définie comme un processus qui peut mener à l'intégration ou au rejet - des minorités hispanophones aux ÉtatsUnis et des communautés musulmanes en Europe. Partant du constat que les groupes dominants dans chaque contexte se définissent partiellement en opposition à la langue espagnole et à l'Islam, Zolberg a exploré dans quelle mesure les sociétés d'accueil permettent l'estompement ou le repositionnement (vers l'inclusion ou vers l'exclusion) de la frontière entre "nous » et « eux ». Il a observé qu'en Europe, même aux Pays-Bas, réputés tolérants envers les différences religieuses, une certaine accommodation institutionnelle vis-à-vis de l'Islam a déclenché des réactions négatives et un repositionnement de la frontière vers une identité chrétienne. Pour Zolberg, l'inclusion 
des « Latinos » hispanophones aux États-Unis serait au contraire facilitée par le fait que la maîtrise de la langue anglaise n'oblige pas l'abandon de la langue maternelle et n'exige aucun comportement spécifique, à la différence des religions monothéistes. Les États-Unis seraient d'ailleurs caractérisés par une capacité exceptionnelle d'adaptation à la diversité religieuse, dont un exemple éclairant se trouve dans le règlement de parking « multiculturel » de la ville de New York. À l'inverse d'un article publié il y a quinze ans intitulé "Why Islam is Like Spanish », Zolberg a souligné ainsi les différences entre ces deux groupes minoritaires dans leurs contextes d'accueil.

Les remarques du conseil scientifique à la fin du colloque ont évoqué, dans un premier temps, les difficultés majeures posées par un colloque international consacré à un sujet aussi large que les migrations. Le temps manquait parfois pour entamer une analyse théorique des données, ce qui a fait obstacle à la comparaison des expériences. Fernando Devoto a notamment questionné la représentativité des études de cas présentées : à quel point les conclusions sont-elles généralisables? Quel est le poids relatif des différents phénomènes sociaux qui provoquent ou structurent certaines migrations ? Ramón Gutierrez, pour sa part, a souligné des aspects plus conflictuels des migrations qui étaient largement absents des interventions, dont les confrontations avec le racisme, les tensions religieuses, les luttes pour l'accès à la citoyenneté et surtout les pratiques gouvernementales de détention et d'expulsion.

Le colloque s'est achevé sur un débat sur la nécessité d'une typologie plus formalisée pour encadrer l'étude des migrations, sujet interdisciplinaire par excellence. Si ce champ de recherche a fait preuve de maturité en abandonnant les vielles notions d'assimilation et d'acculturation qui dominaient autrefois les analyses, les études migratoires demeurent "émergentes ", et la construction de schémas est toujours en cours. Une "remise en ordre " des catégories d'analyse est-elle nécessaire afin de faciliter la communication entre les disciplines? Ou alors un glissement constant entre les catégories aide-t-il à dépasser des cadres stériles et des théories inadéquates qui peuvent enfermer la recherche? Quoi qu'il en soit, les participants se sont accordés pour affirmer que la légitimité de l'enjeu migratoire dans le monde universitaire et audelà est aujourd'hui résolument acquise.

11 Voir le programme complet du colloque sur le site web dédié: https:// sites.google.com/site/colloqueida2012/.

\section{INDEX}

Thèmes : Actualité de la recherche

\section{AUTEUR}

\section{HILARY SANDERS}

Université Lumière Lyon 2 